\title{
A Theory of the Under-water Unit with a Slow-leak for Wave Recorders*
}

\author{
Kantaro WATANABE ${ }^{* *}$
}

\begin{abstract}
Although the structure of the under-water unit with a slow-leak has been described in many papers, it appears that the theoretical analysis for the characteristics of the slow-leak itself has not yet been mentioned. The present paper is an attemt to give a method designing under-water unit with a slow-leak based upon a thoretical consideration. Here are illustrated some examples of the response curves drawn by introducing the slow-leak constant.
\end{abstract}

\section{Introduction}

Numerous wave measurements are made by means of pressure recorders placed at some depths below ocean surface. The recorder of this type generally consists of an under-water unit installed at bottom or some depth and electrically recording device on land or vessel. The pressure fluctuations at bottom caused by the passage of waves overhead compress or expand the bellows of the under-water unit, and the movements of bellows are converted into electrical signals with adequate device. The measurements of wind waves and swells proper are effected by an under-water unit usually incorporated with a slow-leak mechanism filtering long period fluctuations of pressure other than wind waves and swells, which might scale out and destroy. The structure of the under-water unit with a slow-leak has hitherto been discussed by numerous authors (Folsom, 1949; KLEBBA, 1954 ; Munk, 1947), but it seems that the characteristics of the slow-leak itself have not yet been theoretically analysed. The present paper aimed at to treat the problem giving a method of design.

\section{Theory}

A typical construction of the under-water unit is schematically shown in Fig. $1 . R$ is an air resirvoir which communicates through a capillary tube $L$ with the outside of the reservoir. Sometimes other devices have been used instead in many prac-

\footnotetext{
* Received Feb. 8, 1956.

* Central Metorological Observatory, Tokyo
}

tical recorders, but the capillary tube is a representative in the principle. $B_{i}$ and $B_{0}$ are sensing bellows placed inside and outside the reservoir respectively, which are tightly connected with each other by a rod. Therefore, this rod moves upwards or downwards according to the difference of pressures idside and outside. And $E$ is an electrical converter which converts the movements of the rod into electrical signals.

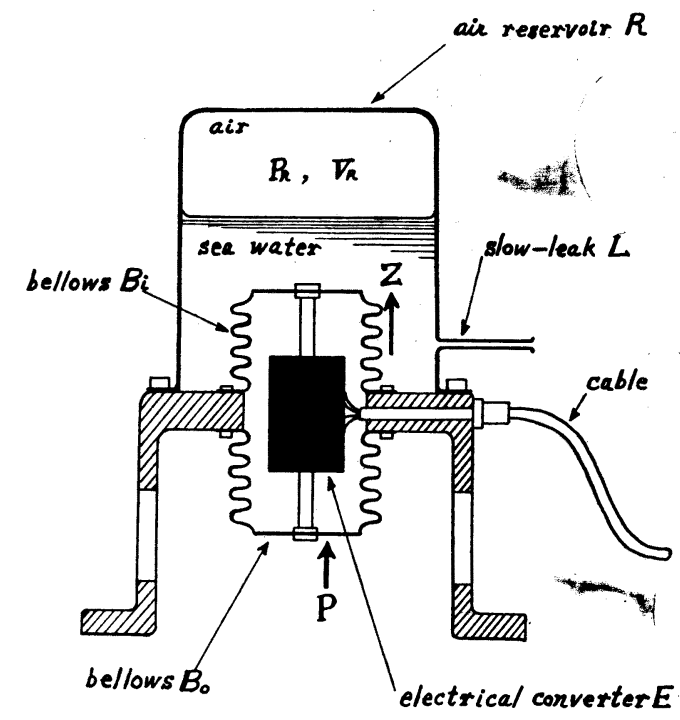

Fig. 1 Schematic diagram of the under-water unit with a slow-leak

Let $P_{R}$ and $V_{R}$ be the pressure and volume of the air of the reservoir respectively, $l$ and $d$ the length and diameter of the capillary tube respectively. Further let $\boldsymbol{B}$ be the effective area of bellows, $B_{i}$ and $B_{o}$ having equal dimensions. If the ambient 
water pressure of the under-water unit is $P$, and the travel of the rod is $Z$, the relation between $P$ and $Z$ is

$$
W Z=B\left(P-P_{R}\right) \text {, }
$$

where $W$ is the additive elastic constant of the bellows system. The water flowing through the capillary into the reservoir during the time interval $d t$ is

$$
d Q=m\left(P-P_{R}\right) d t,
$$

where $m$ is Poiseuille's constant for the capillary. Therefore, $\mu$ standing for the coefficient of viscosity of sea water,

$$
m=\frac{\pi d^{4}}{8 \pi l} \text {. }
$$

At the same time, the volume $d Q$ can be written as

$$
d Q=-d V_{R}-B d Z \text {. }
$$

As the change can be assumed isothermal, we have

$$
\begin{gathered}
P_{R} V_{R}=P^{\prime} V^{\prime}, \\
\therefore \quad \frac{d V_{R}}{d t}=-\frac{V^{\prime}}{P^{\prime}} \frac{d P_{R}}{d t},
\end{gathered}
$$

where $P^{\prime}$ is the mean air pressure inside the reservoir placed at the given depth, $V^{\prime}$ is the air volume inside the reservoir at the pressure $P^{\prime}$. Neglecting the inertia effect of the bellows and rod, we get as the first approximation of the above discussion

where

$$
\dot{Z}+a Z-b \dot{P}=0,
$$

$$
\left.\begin{array}{l}
a=\begin{array}{c}
m W P^{\nu} \\
V^{\prime} W+P^{\prime} B^{2} \\
V^{\prime} B \\
V^{\prime} W+P^{\prime} B^{2}
\end{array}
\end{array}\right\}
$$

If $Z=O$ initaially, the solution of $(6)$ is

$$
Z=b e^{-a t} \int_{0}^{t} e^{a t} \frac{d P}{d t} d t .
$$

Considering the pressure fluctuation to be sinusoidal, we put

$$
\begin{aligned}
P & =p_{0}+p \sin \omega t, \\
\therefore \quad \dot{P} & =\omega p \cos \omega t .
\end{aligned}
$$

Thence, we have

$$
\begin{aligned}
Z= & \frac{ \pm b p}{\sqrt{1+\tan ^{2} \delta}} \sin (\omega t+\delta) \\
& -\frac{\tan \delta}{1+\tan ^{2} \delta} b p e^{-a t},
\end{aligned}
$$

where $\delta$ is the phase difference defined as

$$
a / \omega=\tan \delta \text {. }
$$

After a sufficient time lapse, the second term of (9) vanishes entirely, and the solution becomes

$$
Z \approx \underset{\sqrt{1+\tan ^{2} \delta}}{ \pm \sin (\omega t+\delta) .}
$$

Here the bellows and rod can be seen to displace sinusoidally with time. We put hereon for convinience sake

$$
z \quad \frac{b p}{\sqrt{1+\tan \delta^{2}}} .
$$

Substituting the frequency $\omega$ by the period $T$, the relation between the amplitude $Z$ of the bellows system and $T$ can be written as

$$
\frac{z}{p} / b=c \begin{gathered}
1 \\
\sqrt{ } 1+T^{2} / \lambda^{2}
\end{gathered}
$$

where $\lambda$ is defined as

$$
\lambda 2 \pi / a,
$$

which is to be called the 'slow-leak constant'. And the phase difference is given by

$$
\delta=\tan ^{-1} \frac{T}{\lambda} .
$$

\section{Response Curves}

The equation (11) shows that the response $z / p$ or the ratio of the amplitude $z$ of the bellows system to the pressure amplitude $p$, changes with the period $T$ of the water pressure and the slow-leak constant $\lambda$. Figs. 2 and 3 represent the relations (11) and (13) respectively.

In Fig. 2, we can understand the response $z / p$ is large for shorter periods and small for longer periods for a given value of $\lambda$. This fact shows that the slow-leak behaves like a filter to the pressure fluctuations due to ocean waves. And in the same figure we see that the response is also a function of the slowleak constant $\lambda$. From curves illustrated therein the most suitable value of $\lambda$ can be selected for the periods of waves to be measured.

For example, if such wave recorder is to measure only winds waves and swells of periods less than 20 seconds, and be possibly indifferent to longer-period fluctuations, we 


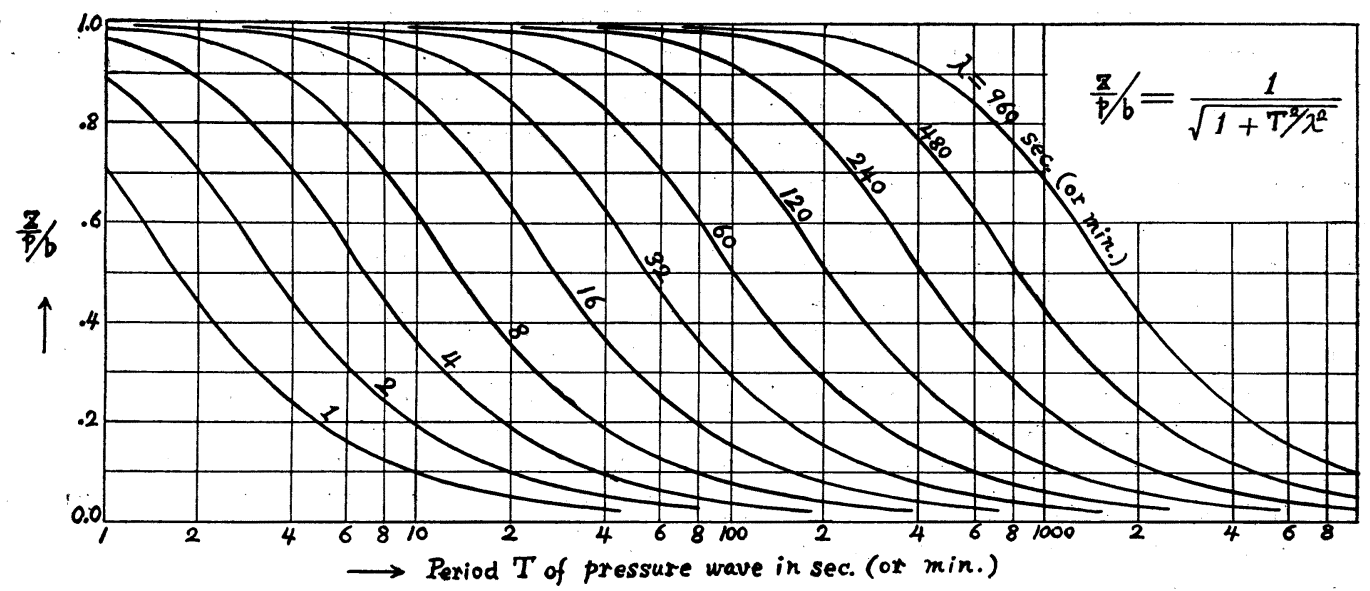

Fig. 2. Response curves

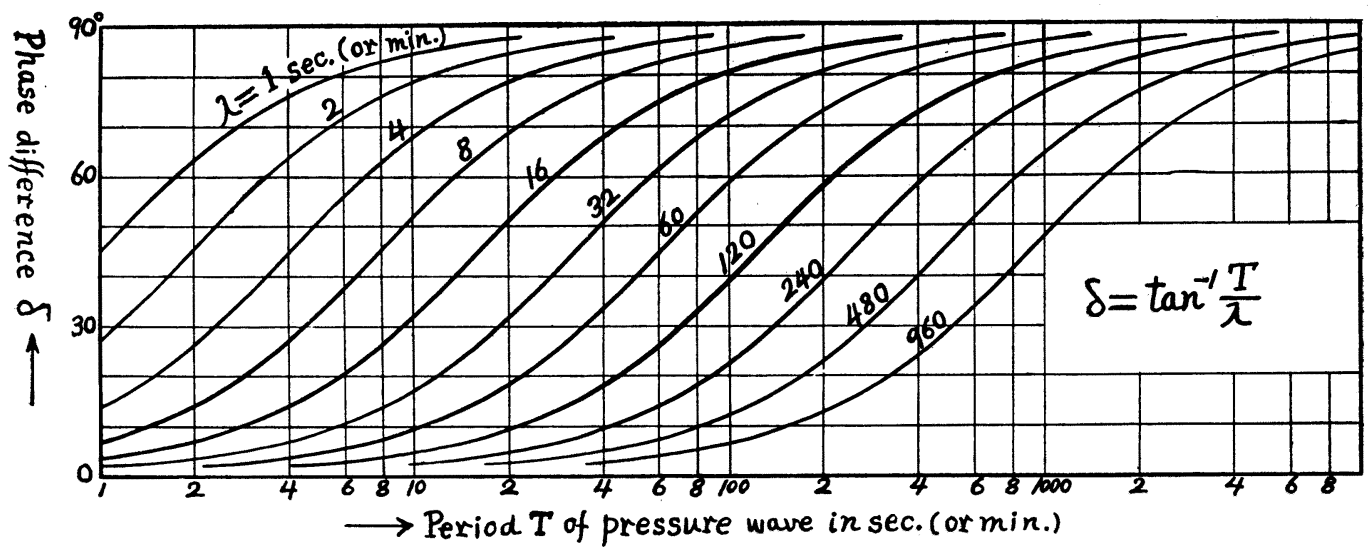

Fig. 3 Phase difference

can find from Fig. 1 that the appropriate slow-leak constant $\lambda$ should be in the vicinity of $60 \mathrm{sec}$. And further it is obvious that the value of the response will be 0.9 or more for period $T$ less than $20 \mathrm{sec}$. and 0.3 or less for $T$ larger than $7 \mathrm{~min}$. Then the recorder will register only ordinary wind waves and swells with its full sensitivity, and the scaling out and destruction due to the large fluctuations of sea level caused by tides and other effects, can safely be avoided.

Once the value of $\lambda$ be selected, the value of $a$ can be determined by the equation (12), and Poiseuille's constant $m$ im (3) can be calculated in the following way. For this purpose we must first give the respective value of $P^{\prime}, V^{\prime}$ and $B, W$, which in turn determine the value of $b$ according to the equation (7).
In the range of period $T$ to be measured the denominator of the right-hand side of (11) is nearly unity, and $z / p$, viz., the maximum sensitivity attainable for every variation of $T$, is seen to be here almost equal to $b$. In (7), $P^{\prime}$ and $V^{\prime}$ are respectivity the mean pressure and mean volume of the air inside the reservoir placed at the given depth, denoted $h$. If we put $P_{0}$ and $V_{0}$ for the values $P^{\prime}$ and $V^{\prime}$ respectively at the surface, the equation connecting $P_{0}$ and $V_{0}$ with $P^{\prime}$ and $V^{\prime}$ is clearly

$$
P^{\prime} V^{\prime}=P_{0} V_{0} \text {. }
$$

Therefore, using $V_{0}$ for $V^{\prime}$ in (7), we get

$$
b=\frac{B V_{0}}{V_{6} W+B^{2} P^{2} / P_{0}} .
$$

Measuring $h$ in metres we can express $P \approx$ $(1+0.1 h) P_{\theta}$, and we get 


$$
b=\frac{B V_{0}}{V_{0} W+(1+0.1 h)^{2} P_{0} B^{2}},
$$

where $P_{0}$ natually equals to the standard atmospheric pressure at the surface and $V_{0}$ should be taken to be nearly equal to the capacity of the air reservoir. Thus $B$ and $W$ of the bellows being assumed for the design, the maximum sensitivity $b$ for a given bepth $h$ (metres), can be calculated or inversely the volume $V_{0}$ of the air reservoir for a desired sensitivity $b$. In this way the value of PoISEUILLE's constant $m$ will accordingly be determined in the equation (7) and, if necessary, the dimension of the capillary tube will be designed in equation $(3)$, or as its counterpart, other slow-leak devices may similarily be effected.
Acknowledgement-The author wishes to thank Dr. M. SANuKi (Central Meteorological Observatory) for his constant encouragement and kind advice.

\section{References}

Folsom, R. G., 1949:

"Measurement of ocean waves", Trans. Amer. Geophys. Union, 30, 691.

KLEBBA, A. A., 1954 :

"Details of shore-based wave recorder and ocean wave analyzer", Ann. N. Y., Acad. Sci., 51, art. $3,343$.

MunK, W. H., 1947:

"Tracking storm by forerunners of swell", Jour. Meteor., 4-6, 45. 